\title{
Exosome-associated Mitochondrial DNA is Elevated in Patients with ME/CFS and Stimulates Human Cultured Microglia to Secrete IL-1 $\beta$
}

Theoharis C Theoharides ( $\nabla$ theoharis.theoharides@tufts.edu )

Tufts University School of Medicine https://orcid.org/0000-0002-1598-460X Irene Tsilioni

Tufts University School of Medicine

Benjamin Natelson

Icahn School of Medicine at Mount Sinai

Short report

Keywords: Myalgic Encephalomyelitis/Chronic Fatigue Syndrome (ME/CFS), sleep disturbances, malaise , cognitive problems

Posted Date: January 28th, 2021

DOI: https://doi.org/10.21203/rs.3.rs-154011/v1

License: (c) (i) This work is licensed under a Creative Commons Attribution 4.0 International License. Read Full License 


\section{Abstract}

Background: Myalgic Encephalomyelitis/Chronic Fatigue Syndrome (ME/CFS) is a debilitating disease that presents with fatigue, sleep disturbances, malaise and cognitive problems. The pathogenesis of $\mathrm{ME} / \mathrm{CFS}$ is presently unknown and serum levels of potential biomarkers have been inconsistent.

Methods: Exosomes were purified from serum obtained from patients with ME/CFS before and after exercise and their content of mitochondrial DNA (mtDNA) was determined by quantitative PCR.

Exosomes from both patients and controls were incubated with cultured human microglia and release of interleukin-1 beta (IL-1 $\beta$ ) was measured by ELISA.

Results: Here we show that serum mtDNA, associated with exosomes, is increased in ME/CFS after exercise. Moreover, exosomes isolated from patients with ME/CFS stimulate significant secretion of IL-1 $\beta$ from cultured human microglia.

Conclusion: These results provide evidence for a potential novel pathogenetic factor and target for treatment of ME/CFS.

\section{Introduction}

Myalgic encephalomyelitis/Chronic fatigue syndrome CFS (ME/CFS) is a chronic, debilitating disease affecting $1 \%$ of the US population ${ }^{1}$ with an economic burden of about $\$ 20$ billion. ${ }^{2}$ The prevalence of $\mathrm{ME} / \mathrm{CFS}$ is about $0.3 \%$ in the USA, ${ }^{3}$ occurs predominantly in women, and is characterized by disabling fatigue. ${ }^{4-8}$ However, the core symptoms of fatigue, sleep problems and cognitive difficulties exist across other comorbidities, such as fibromyalgia syndrome (FMS). 6, 9-12

The pathogenesis of ME/CFS is still unknown, but patients with ME/CFS showed difficulties in cognitive performance, ${ }^{13}$ and approximately half developed their illness following a sudden, influenza-like illness. ${ }^{11} \mathrm{ME} / \mathrm{CFS}$ may be an autoimmune disease ${ }^{14,15}$ involving neuroinflammation. ${ }^{16-19}$ Even though serum pro-inflammatory cytokine levels have been reported to be increased in ME/CFS patients ${ }^{20}$ other studies using sedentary controls have not supported such findings either before or after stress induced by exercise or sleep deprivation. ${ }^{6,21}$ As a result, it was suggested that ME/CFS may involve some dysfunction in the brain. ${ }^{22}$ We proposed that local inflammation in the hypothalamus could dysregulate homeostasis. ${ }^{23}$

Extracellular vesicles (EVs) are membrane-bound vesicles ranging from 30-10,000 nm diameter, with exosomes considered the subfraction of 30-300 nm in diameter. ${ }^{24} \mathrm{EVs}$ have been shown to carry different types of cargo including microRNAs, proteins and lipids with the potential to alter pathophysiological processes. ${ }^{25}$ In particular, exosomes have been implicated in brain disorders, ${ }^{26,27}$ but their potential role in ME/CFS has not been adequately investigated. 
Here we show that serum mitochondrial DNA (mtDNA), associated with exosomes, is increased only in $\mathrm{ME} / \mathrm{CFS}$ patients as compared to healthy controls, and stimulates cultured human microglia to secrete interleukin-1 beta (IL-1b).

\section{Methods}

\section{Exosome Isolation:}

Total EVs were isolated using the exoEasy Maxi Kit (Qiagen, Valencia, CA) from $1 \mathrm{~mL}$ of serum. Prefiltered serum ( $0.8 \mu \mathrm{m}$ syringe filter) were mixed with Buffer XBP and were bounded to an exoEasy membrane affinity spin column. The bound EVs were washed with Buffer XWP, were eluted with $400 \mu \mathrm{l}$ Buffer XE (an aqueous buffer containing primarily inorganic salts) and were then ready to use for further analysis. The reason we used this commercialy available exosome purification kit is due to the limited amount of biological samples ( $2 \mathrm{~mL}$ serum from each ME/CFS patient and healthy control) available in our possession.

\section{BCA assay:}

The concentration of total protein was quantified by the bicinchoninic acid (BCA) assay (Thermo Fisher Inc., Rockford, IL) using bovine serum albumin (BSA) as standard.

\section{Electron microscopy:}

A drop of isolated serum-derived total EVs suspended in Buffer XE was deposited on Formvar-carboncoated electron microscopy grids, fixed as above, immunolabelled and stained using the method as described before. All samples were analyzed at Harvard Medical School's Electron Microscopy (EM) Core Facility by Ms. Maria Ericsson, Manager of the Harvard Medical School EM Facility, using the Tecnai G2 Spirit BioTWIN transmission electron microscope (TEM).

\section{Mitochondrial DNA:}

Total DNA was extracted from exosomes using Qiagen DNA Micro extraction kit (Qiagen, CA). Mitochondrial specific DNA for 7S (mt-7S) was quantified by RT-qPCR using Taqman gene expression assays (Mt-7S: Hs02596861_s1; GAPDH: Hu, VIC, TAMRA, Applied Biosystems, Carlsbad, CA). Samples were run at 45 cycles using Applied Biosystems 7300 Real-Time PCR System. GAPDH DNA was used to exclude any genomic "contamination."

\section{Cell culture:}


SV40 immortalized human adult microglia, frozen in the M1 pro-inflammatory state, were purchased from Applied Biological Materials Inc., (ABM, Vancouver, Canada). Microglia were cultured in Prigrow III medium (ABM) supplemented with $10 \% \mathrm{FBS}$ and $100 \mathrm{U} / \mathrm{mL}$ penicillin/streptomycin using BD PureCoat ECM Mimetic Cultureware Collagen I peptide plates (Thermo Sci.).

\section{Cell viability assay:}

Cell viability was measured by Trypan blue (1\%) exclusion.

\section{Mediator assay:}

IL-1b was assayed using commercial ELISA kits from R\&D Systems (Minneapolis, MN). Control cells were treated with equal volume of culture medium only.

\section{Power analysis and statistics:}

Primary objective: Content of serum EV-mtDNA from ME/CFS patients. The required sample size to observe a difference of $30 \%$ in EV-associated mtDNA between ME/CFS patients and controls at $5 \%$ significance level with a power of $80 \%$ is at least 25 subjects/group. Correlations between mtDNA and either subgroup were determined using the Spearman rank correlation test. Comparisons with control were done using either parametric t-test for independent samples or Mann-Whitney non-parametric test depending on normality of distribution to be checked with the Shapiro-Wilk's test. Comparisons between the groups were done with ANOVA and Wilcoxon post-hoc paired rank sum test.

\section{Results}

\section{Serum levels of exosome-associated mtDNA in ME/CFS patients.}

Exosomes were isolated and characterized by electron microscopy confirming their spherical shape covered by a membrane (Fig $1 \mathrm{~A}$ ) and then by Western blot analysis. Total exosome-associated protein was lower in ME/CFS patients as compared to controls and was even lower after exercise (Fig. 1B).

The serum level of mtDNA (7S) was increased only after exercise in ME/CFS patients (Fig. 2). Moreover, the ratio of mtDNA to that of exosome-protein was significantly increased only in ME/CFS as compared to controls.

\section{Exosomes from ME/CFS patients activate human microglia.}


Total exosomes isolated from patients with ME/CFS stimulate significant release of IL-1 $\beta$ from cultured human microglia (Fig. 3). These results were obtained using mtDNA pooled from 5 subjects from either control or ME/CFS subjects and not with mtDNA from each individual subject.

\section{Discussion}

We isolated total exosomes (30-100 nm fin diameter) from serum samples obtained from ME/CFS patients and controls as we reported previously. ${ }^{28}$ Interestingly, we reported less total EV-associated protein obtained from serum of ME/CFS patients than controls unlike two other studies that reported no significant difference in EV yields obtained from plasma of ME/CFS patients and controls. ${ }^{29,} 30$ In contrast, two other studies reported a significant increase in EV content in the serum of ME/CFS patients and controls. ${ }^{31,32}$ These apparent differences may depend on the activity status of the controls, use of serum or plasma, as well as method of EV isolation and purification, thus highlighting the need for standardization for future studies.

Exosomes are EVs with a size range of $30-100 \mathrm{~nm}$ in diameter and may carry different cargo than larger EVs (100-1000 nm in diameter). ${ }^{24} \mathrm{EVs}$ have been implicated in brain disorders, ${ }^{26,27}$ but their role in $\mathrm{ME} / \mathrm{CFS}$ had not been adequately investigated. EVs have been isolated from ME/CFS patients and characterized especially for their content of microRNA. ${ }^{30}$ Another study investigated the cytokine profile of plasma and MEs isolated from plasma of ME/CFS patients and reported no significant differences. ${ }^{29}$ Hence, the amount of circulating cytokines whether free or EV=-associated may not be important, as compared to the ability of EVs to potentially stimulate the release of pro-inflammatory cytokines from microglia in the brain.

The source of exosomes in ME/CFS patients in not presently known. One possibility is that they derive from mast cells ${ }^{33,34}$ either in the hypothalamus where they are particularly abundant ${ }^{35,36}$ perivascularly in close proximity to neurons ${ }^{36,37}$ or from peripheral mast cells and enter the brain by crossing the bloodbrain barrier (BBB), which is known to be disrupted in neuropsychiatric disorders. ${ }^{38,39}$ Mast cells and their mediators have been implicated in diseases comorbid with ME/CFS. ${ }^{40}$ Moreover, mast cells are increased in the skin of patients with ME/CFS, ${ }^{41,42}$ who also show increased skin hypersensitivity. ${ }^{43}$ Hyper-responsiveness of the bronchi, implying activation of mast cells, has also been noted in ME/CFS patients. ${ }^{44}$ Activated mast cells could release additional mediators contributing to ME/CFS symptoms, 45,46 or mtDNA. ${ }^{47}$ The possible mast cell source of the exosomes could be determined in the future by Western blot analysis of the presence in exosomes of the mast cell-specific surface markers (FceRI, MRGX2 and CD-117). ${ }^{34}$

Here we show that serum mitochondrial DNA (mtDNA), associated with exosomes, is increased after exercise in patients with ME/CFS as compared to unrelated, healthy controls. Moreover, we report that exosomes containing mtDNA from patients with ME/CFS stimulate cultured human microglia to secrete IL-1b. We had reported that exosomes purified from serum from children with autism spectrum disorder 
(ASD) contained mtDNA and stimulated human microglia to secrete IL-1b. ${ }^{28}$ This key pro-inflammatory cytokine has been implicated in fatigue ${ }^{48}$ and in a rat model of CFS. ${ }^{49}$ We had also reported that extracellular mtDNA is increased in the serum of children with ASD. ${ }^{50}$

Mitochondrial DNA could act as an "innate pathogen" leading to a localized auto-inflammatory response in the hypothalamus. ${ }^{51-54}$ Analysis of the mitochondrial genome in ME/CFS cases indicated that individuals with a certain haplotype were more likely to exhibit certain neurologic symptoms, but there was no association with either susceptibility ${ }^{55}$ or disease severity. ${ }^{56}$ An unusual pattern of mtDNA deletions was reported in the skeletal muscle of one patient with ME/CFS. ${ }^{57}$ Cellular bioenergetics has been reported to be impaired in patients with ME/CFS ${ }^{58}$ and salivary mtDNA was found to be decreased in subjects with fatigue. ${ }^{59}$ Even though mtDNA was shown to be neurotoxic in rat brain slices, ${ }^{60-62}$ the mtDNA molecular patterns $\mathrm{N}$-formyl peptides and cardiolipin did not stimulate IL-6 or TNF release from HMC-3 microglia ${ }^{63}$ implying that the entire mtDNA may be required for stimulation.

There are a number of limitations in this study. First, the number of subjects analyzed was small. Pathogenetic changes in ME/CFS patients may only occur in the brain, especially the hypothalamus, in which case we will have to await the availability of brain tissue to carry out analysis of the proposed biomarkers, as we recently reported for autism spectrum disorder. ${ }^{64}$

\section{Conclusion}

These results provide evidence for a novel pathogenetic factor involving exosome-associated mtDNA stimulating microglia release of IL-1b that could lead to the development of new treatments for ME/CFS.

\section{Abbreviations}

ASD: Autism spectrum disorder

BBB: Blood-brain barrier (BBB),

BCA: Bicinchoninic acid

$\mathrm{CRH}$ : Corticotropin-releasing hormone

EVs: Extracellular vesicles

FMS: Fibromyalgia syndrome

IL-1b: Interleukin-1beta

mtDNA: Mitochondrial DNA

ME/CFS: Myalgic Encephalomyelitis/Chronic Fatigue Syndrome 


\section{Declarations}

\section{Ethics approval and consent to participate}

All patients had participated in a previous study that had been approved by the appropriate Institutional Review Board of UMDNJ and all patients had provided written informed consent. For the present study, all sera used were from de-identified patients.

\section{Consent for publication}

Not applicable

\section{Availability of data and materials}

The datasets used and analyzed during the current study are available upon reasonable request.

\section{Competing interests}

The authors declare that they have no competing interests.

\section{Funding}

Funded in part by the Ramsay Award from Solve ME/CFS Foundation.

\section{Authors' Contributions}

TCT and IT conceived the concept. IT performed the experiments, the data collection and their analysis. BN provided the patients samples and discussed the results and their implications. TCT prepared the manuscript. All authors read and approved the manuscript.

\section{Acknowledgements}

We thank the Solve ME/CFS Foundation for the Ramsay Award.

\section{References}


1. Cortes RM, Mastronardi C, Silva-Aldana CT, rcos-Burgos M, Lidbury BA. Myalgic Encephalomyelitis/Chronic Fatigue Syndrome: A Comprehensive Review. Diagnostics (Basel) 2019; 9(3).

2. Jason LA, Benton MC, Valentine L, Johnson A, Torres-Harding S. The economic impact of ME/CFS: individual and societal costs. Dyn Med. 2008;7:6.

3. Jason LA, Richman JA, Rademaker AW, et al. A community-based study of chronic fatigue syndrome. Arch Intern Med. 1999;159(18):2129-37.

4. Van't LM, Zielhuis GA, van der Meer JW, Verbeek AL, Bleijenberg G. Fatigue and chronic fatigue syndrome-like complaints in the general population. Eur J Public Health. 2010;20(3):251-7.

5. Griffith JP, Zarrouf FA. A systematic review of chronic fatigue syndrome: don't assume it's depression. Prim Care Companion J Clin Psychiatry. 2008;10(2):120-8.

6. Natelson BH. Myalgic Encephalomyelitis/Chronic Fatigue Syndrome and Fibromyalgia: Definitions, Similarities, and Differences. Clin Ther. 2019;41(4):612-8.

7. Jason LA, Corradi K, Torres-Harding S, Taylor RR, King C. Chronic fatigue syndrome: the need for subtypes. Neuropsychol Rev. 2005;15(1):29-58.

8. Anderson VR, Jason LA, Hlavaty LE, Porter N, Cudia J. A review and meta-synthesis of qualitative studies on myalgic encephalomyelitis/chronic fatigue syndrome. Patient Educ Couns. 2012;86(2):147-55.

9. Wessely S, Nimnuan C, Sharpe M. Functional somatic syndromes: one or many? Lancet. 1999;354(9182):936-9.

10. Barsky AJ, Borus JF. Functional somatic syndromes. Ann Intern Med. 1999;130(11):910-21.

11. Ciccone DS, Natelson BH. Comorbid illness in women with chronic fatigue syndrome: a test of the single syndrome hypothesis. Psychosom Med. 2003;65(2):268-75.

12. Natelson BH, Lin JS, Lange G, Khan S, Stegner A, Unger ER. The effect of comorbid medical and psychiatric diagnoses on chronic fatigue syndrome. Ann Med. 2019;51(7-8):371-8.

13. Cook DB, Nagelkirk PR, Peckerman A, Poluri A, Mores J, Natelson BH. Exercise and cognitive performance in chronic fatigue syndrome. Med Sci Sports Exerc. 2005;37(9):1460-7.

14. Theoharides TC, Weinkauf C, Conti P. Brain cytokines and neuropsychiatric disorders. J Clin Psychopharmacol. 2004;24(6):577-81.

15. Sotzny F, Blanco J, Capelli E, et al. Myalgic Encephalomyelitis/Chronic Fatigue Syndrome - Evidence for an autoimmune disease. Autoimmun Rev. 2018;17(6):601-9.

16. Bansal AS, Bradley AS, Bishop KN, Kiani-Alikhan S, Ford B. Chronic fatigue syndrome, the immune system and viral infection. Brain Behav Immun. 2012;26(1):24-31.

17. Dietert RR, Dietert JM. Possible role for early-life immune insult including developmental immunotoxicity in chronic fatigue syndrome (CFS) or myalgic encephalomyelitis (ME). Toxicology. 2008;247(1):61-72. 
18. Bower JE. Fatigue, brain, behavior, and immunity: summary of the 2012 Named Series on fatigue. Brain Behav Immun. 2012;26(8):1220-3.

19. Nakatomi Y, Mizuno K, Ishii A, et al. Neuroinflammation in Patients with Chronic Fatigue Syndrome/Myalgic Encephalomyelitis: An 11C-(R)-PK11195 PET Study. J Nucl Med. 2014;55(6):945-50.

20. Maes M, Twisk FN, Ringel K. Inflammatory and cell-mediated immune biomarkers in myalgic encephalomyelitis/chronic fatigue syndrome and depression: inflammatory markers are higher in myalgic encephalomyelitis/chronic fatigue syndrome than in depression. Psychother Psychosom. 2012;81(5):286-95.

21. Nakamura T, Schwander S, Donnelly R, et al. Exercise and sleep deprivation do not change cytokine expression levels in patients with chronic fatigue syndrome. Clin Vaccine Immunol. 2013;20(11):1736-42.

22. Natelson BH. Brain dysfunction as one cause of CFS symptoms including difficulty with attention and concentration. Front Physiol. 2013;4:109.

23. Hatziagelaki E, Adamaki M, Tsilioni I, Dimitriadis G, Theoharides TC. Myalgic Encephalomyelitis/Chronic Fatigue Syndrome-Metabolic Disease or Disturbed Homeostasis due to Focal Inflammation in the Hypothalamus? J Pharmacol Exp Ther. 2018;367(1):155-67.

24. Raposo G, Stoorvogel W. Extracellular vesicles: exosomes, microvesicles, and friends. J Cell Biol. 2013;200(4):373-83.

25. Yang B, Chen Y, Shi J. Exosome Biochemistry and Advanced Nanotechnology for Next-Generation Theranostic Platforms. Adv Mater. 2019;31(2):e1802896.

26. Tsilioni I, Panagiotidou S, Theoharides TC. Exosomes in neurologic and psychiatric disorders. Clin Ther. 2014;36(6):882-8.

27. Kawikova I, Askenase PW. Diagnostic and therapeutic potentials of exosomes in CNS diseases. Brain Res. 2014;1617:63-71.

28. Tsilioni I, Theoharides TC. Extracellular vesicles are increased in the serum of children with autism spectrum disorder, contain mitochondrial DNA, and stimulate human microglia to secrete IL-1beta. J Neuroinflammation. 2018;15(1):239.

29. Giloteaux L, O'Neal A, Castro-Marrero J, LeVine SM, Hanson MR. Cytokine profiling of extracellular vesicles isolated from plasma in myalgic encephalomyelitis/chronic fatigue syndrome: a pilot study. J Transl Med. 2020;18(1):387.

30. menar-Perez E, Sarria L, Nathanson L, Oltra E. Assessing diagnostic value of microRNAs from peripheral blood mononuclear cells and extracellular vesicles in Myalgic Encephalomyelitis/Chronic Fatigue Syndrome. Sci Rep. 2020;10(1):2064.

31. Eguchi A, Fukuda S, Kuratsune $H$, et al. Identification of actin network proteins, talin-1 and filamin-A, in circulating extracellular vesicles as blood biomarkers for human myalgic encephalomyelitis/chronic fatigue syndrome. Brain Behav Immun. 2020;84:106-14. 
32. Castro-Marrero J, Serrano-Pertierra E, Oliveira-Rodriguez M, et al. Circulating extracellular vesicles as potential biomarkers in chronic fatigue syndrome/myalgic encephalomyelitis: an exploratory pilot study. J Extracell Vesicles. 2018;7(1):1453730.

33. Theoharides TC, Valent P, Akin C. Mast Cells, Mastocytosis, and Related Disorders. N Engl J Med. 2015;373(2):163-72.

34. Kim DK, Cho YE, Komarow HD, et al. Mastocytosis-derived extracellular vesicles exhibit a mast cell signature, transfer KIT to stellate cells, and promote their activation. Proc Natl Acad Sci U S A. 2018;115(45):E10692-701.

35. Theoharides TC, Konstantinidou A. Corticotropin-releasing hormone and the blood-brain-barrier. Front Biosci. 2007;12:1615-28.

36. Rozniecki JJ, Dimitriadou V, Lambracht-Hall M, Pang X, Theoharides TC. Morphological and functional demonstration of rat dura mast cell-neuron interactions in vitro and in vivo. Brain Res. 1999;849:1-15.

37. Dimitriadou V, Rouleau A, Trung Tuong MD, et al. Functional relationships between sensory nerve fibers and mast cells of dura mater in normal and inflammatory conditions. Neuroscience. 1997;77:829-39.

38. Kealy J, Greene C, Campbell M. Blood-brain barrier regulation in psychiatric disorders. Neurosci Lett 2018;133664.

39. Fiorentino M, Sapone A, Senger S, et al. Blood-brain barrier and intestinal epithelial barrier alterations in autism spectrum disorders. Mol Autism. 2016;7:49.

40. Theoharides TC. Atopic conditions in search of pathogenesis and therapy. Clin Ther. 2013;35(5):544-7.

41. Blanco I, Beritze N, Arguelles M, et al. Abnormal overexpression of mastocytes in skin biopsies of fibromyalgia patients. Clin Rheumatol. 2010;29(12):1403-12.

42. Enestrom S, Bengtsson A, Frodin T. Dermal IgG deposits and increase of mast cells in patients with fibromyalgia-relevant findings or epiphenomena? Scand J Rheumatol. 1997;26(4):308-13.

43. Steinberg P, Pheley A, Peterson PK. Influence of immediate hypersensitivity skin reactions on delayed reactions in patients with chronic fatigue syndrome. J Allergy Clin Immunol. 1996;98(6 Pt 1):1126-8.

44. Nijs J, De BP, De MK, et al. Associations between bronchial hyperresponsiveness and immune cell parameters in patients with chronic fatigue syndrome. Chest. 2003;123(4):998-1007.

45. Theoharides TC, Papaliodis D, Tagen M, Konstantinidou A, Kempuraj D, Clemons A. Chronic fatigue syndrome, mast cells, and tricyclic antidepressants. J Clin Psychopharmacol. 2005;25(6):515-20.

46. Theoharides TC, Donelan JM, Papadopoulou N, Cao J, Kempuraj D, Conti P. Mast cells as targets of corticotropin-releasing factor and related peptides. Trends Pharmacol Sci. 2004;25(11):563-8.

47. Zhang B, Asadi S, Weng Z, Sismanopoulos N, Theoharides TC. Stimulated human mast cells secrete mitochondrial components that have autocrine and paracrine inflammatory actions. PloS One. 2012;7(12):e49767. 
48. Roerink ME, van der Schaaf ME, Dinarello CA, Knoop H, van der Meer JW. Interleukin-1 as a mediator of fatigue in disease: a narrative review. J Neuroinflammation. 2017;14(1):16.

49. Yasui M, Menjyo Y, Tokizane K, et al. Hyperactivation of proprioceptors induces microglia-mediated long-lasting pain in a rat model of chronic fatigue syndrome. J Neuroinflammation. 2019;16(1):67.

50. Zhang B, Angelidou A, Alysandratos KD, et al. Mitochondrial DNA and anti-mitochondrial antibodies in serum of autistic children. J Neuroinflammation. 2010;7(1):80.

51. Theoharides TC, Asadi S, Panagiotidou S, Weng Z. The "missing link" in autoimmunity and autism: Extracellular mitochondrial components secreted from activated live mast cells. Autoimmun Rev. 2013;12(12):1136-42.

52. Collins LV, Hajizadeh S, Holme E, Jonsson IM, Tarkowski A. Endogenously oxidized mitochondrial DNA induces in vivo and in vitro inflammatory responses. J Leukoc Biol. 2004;75(6):995-1000.

53. Marques PE, Amaral SS, Pires DA, et al. Chemokines and mitochondrial products activate neutrophils to amplify organ injury during mouse acute liver failure. Hepatology. 2012;56(5):1971-82.

54. Sun S, Sursal T, Adibnia Y, et al. Mitochondrial DAMPs increase endothelial permeability through neutrophil dependent and independent pathways. PloS One. 2013;8(3):e59989.

55. Billing-Ross P, Germain A, Ye K, Keinan A, Gu Z, Hanson MR. Mitochondrial DNA variants correlate with symptoms in myalgic encephalomyelitis/chronic fatigue syndrome. J Transl Med. 2016;14:19.

56. Hanson MR, Gu Z, Keinan A, Ye K, Germain A, Billing-Ross P. Association of mitochondrial DNA variants with myalgic encephalomyelitis/chronic fatigue syndrome (ME/CFS) symptoms. J Transl Med. 2016;14(1):342.

57. Zhang C, Baumer A, Mackay IR, Linnane AW, Nagley P. Unusual pattern of mitochondrial DNA deletions in skeletal muscle of an adult human with chronic fatigue syndrome. Hum Mol Genet. 1995;4(4):751-4.

58. Tomas C, Brown A, Strassheim V, Elson JL, Newton J, Manning P. Cellular bioenergetics is impaired in patients with chronic fatigue syndrome. PLoS ONE. 2017;12(10):e0186802.

59. Shin J, Kim KC, Lee DC, Lee HR, Shim JY. Association between Salivary Mitochondrial DNA Copy Number and Chronic Fatigue according to Combined Symptoms in Korean Adults. Korean J Fam Med. 2017;38(4):206-12.

60. Martin S, Dicou E, Vincent JP, Mazella J. Neurotensin and the neurotensin receptor-3 in microglial cells. J Neurosci Res. 2005;81(3):322-6.

61. Patel AB, Tsilioni I, Leeman SE, Theoharides TC. Neurotensin stimulates sortilin and mTOR in human microglia inhibitable by methoxyluteolin, a potential therapeutic target for autism. Proc Natl Acad Sci U S A. 2016;113:E7049-58.

62. Lauritzen $\mathrm{KH}$, Moldestad O, Eide L, et al. Mitochondrial DNA toxicity in forebrain neurons causes apoptosis, neurodegeneration, and impaired behavior. Mol Cell Biol. 2010;30(6):1357-67.

63. Nasi M, De GA, Bianchini E, et al. Mitochondrial damage-associated molecular patterns stimulate reactive oxygen species production in human microglia. Mol Cell Neurosci. 2020;108:103538. 
64. Tsilioni I, Patel AB, Pantazopoulos H, et al. IL-37 is increased in brains of children with Autism Spectrum Disorder and inhibits human microglia stimulated by neurotensin. Proc Natl Acad Sci U S A. 2019;116(43):21659-65.

\section{Figures}

\section{Figure 1}

A

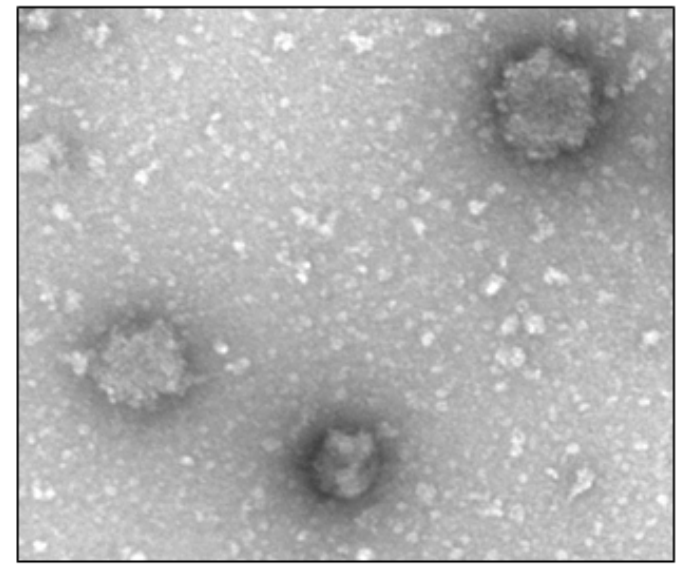

B

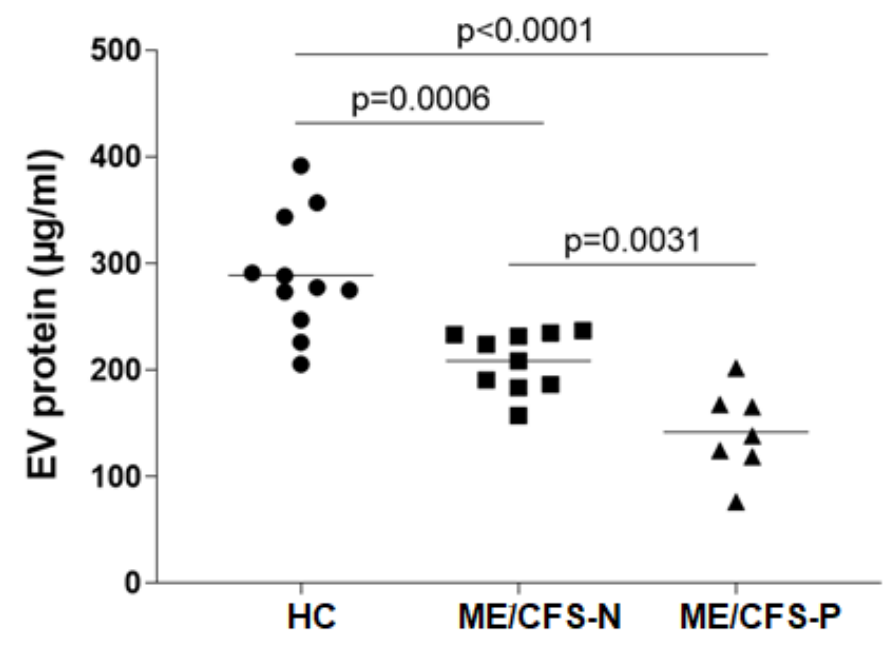

Figure 1

(A) Electron photomicrograph of exosomes. (B) Total serum exosome-associated protein in healthy controls $(\mathrm{HC}), \mathrm{ME} / \mathrm{CFS}$ patients without exercise $(\mathrm{N})$ or past exercise $(\mathrm{P})$. Each dot represents individual subjects. 


\section{Figure 2}

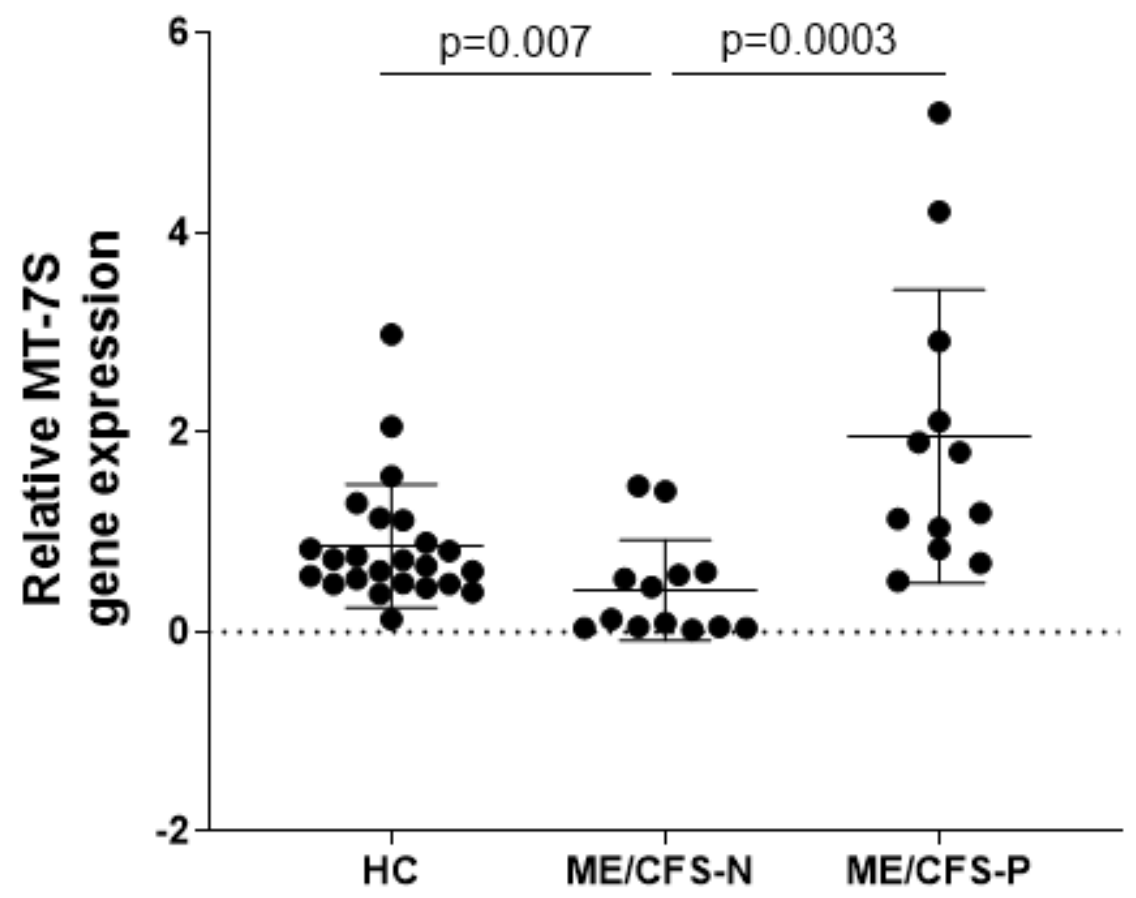

Figure 2

Gene expression of mtDNA (7S) in healthy controls (HC) and ME/CFS patients without exercise (N) or past exercise $(P)$. Each dot represents individual subjects. mtDNA(7S) was normalized to the mean of all control samples. GAPDH was undetectable. 


\section{Figure 3}

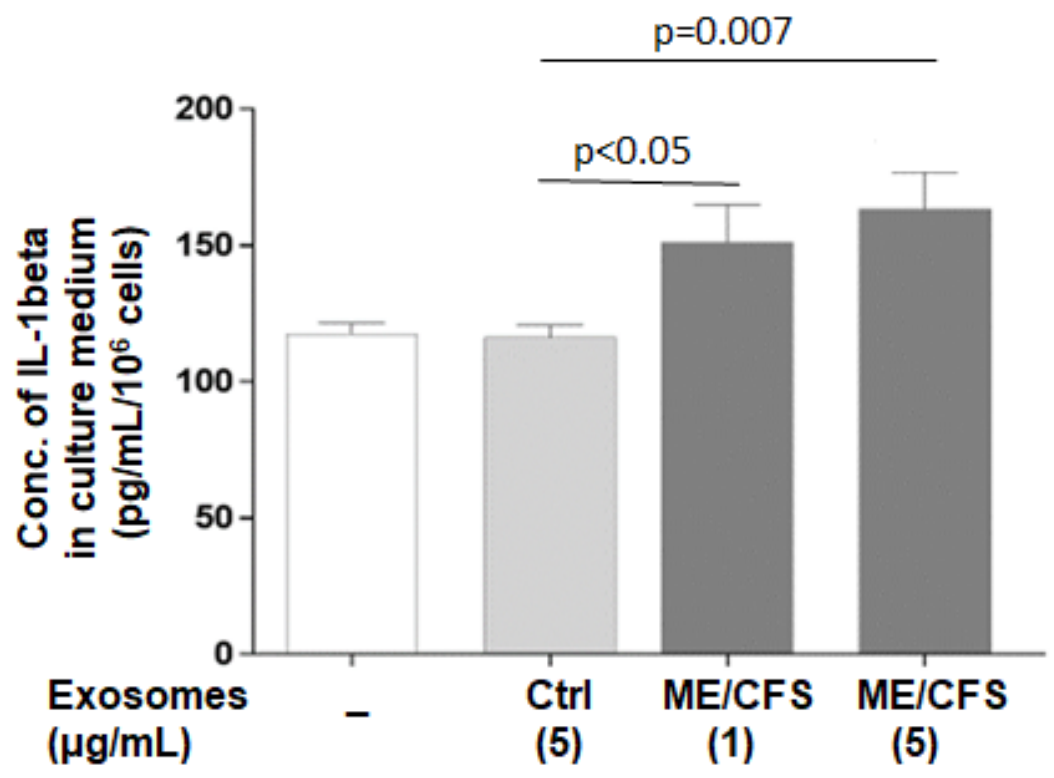

Figure 3

Effect of exosomes on human microglia. Immortalized HM-SV40 microglia (1.0 x 105 cells) were stimulated with exosomes (1 or $5 \mu \mathrm{g} / \mathrm{mL}$ as indicated) obtained and pooled from five subjects from each category (ctrl=control or ME/CFS). Secretion of IL-1 $\beta$ was measured in the supernatant fluid by ELISA $\left(n=3,{ }^{*} p<0.05\right.$ and ${ }^{* * * *} p<0.0001$ compared to controls). 